\title{
EXPLICIT YAMABE FLOW OF AN ASYMMETRIC CIGAR*
}

\author{
ALMUT BURCHARD ${ }^{\dagger}$, ROBERT J. MCCANN ${ }^{\ddagger}$, AND AARON SMITH ${ }^{\S}$ \\ Dedicated to Neil S. Trudinger, in honour of his 65th birthday
}

\begin{abstract}
We consider the Yamabe flow of a conformally Euclidean manifold for which the conformal factor's reciprocal is a quadratic function of the Cartesian coordinates at each instant in time. This leads to a class of explicit solutions having no continuous symmetries (no Killing fields) but which converge in time to the cigar soliton (in two-dimensions, where the Ricci and Yamabe flows coincide) or in higher dimensions to the collapsing cigar. We calculate the exponential rate of this convergence precisely, using the logarithm of the optimal bi-Lipschitz constant to metrize distance between two Riemannian manifolds.
\end{abstract}

Key words. Exact Yamabe flows, Ricci flow, conformally flat non-compact manifold, quadratic conformal factor, cigar soliton, attractor, basin of attraction, rate of convergence, Lyapunov exponent, biLipschitz.

AMS subject classifications. Primary 53C44; Secondary 35K55, 58J35

1. Introduction. We consider explicit solutions to the Yamabe flow on certain asymmetric non-compact manifolds, and analyze their long-term behavior. The manifolds are conformally flat and cigar-shaped, with a positively curved cap and one end. The end is asymptotic to an infinite cylinder, which is symmetric under translation but in general not symmetric under rotation, and whose curvature assumes both positive and negative values. These solutions are interesting both because explicit solutions are rare - especially in the absence of symmetries, and because we are able to compute the rate of symmetrization quite explicitly, using the optimal bi-Lipschitz constant to metrize the distance between two Riemannian manifolds. Though the optimal bi-Lipschitz correspondence remains unknown, we estimate enough about it to complete our rate calculation by studying its behavior on the asymptotically cylindrical ends of our manifolds.

The Yamabe flow was introduced by Hamilton in the late 1980's as a parabolic analogue of the Yamabe problem [12] (see [4]). It deforms a given Riemannian manifold of dimension $n \geq 2$ by evolving its metric $g_{i j}$ according to

$$
\frac{\partial g_{i j}}{\partial t}=-\frac{1}{n-1} R g_{i j}
$$

where $R$ is the scalar curvature. If the manifold is conformally flat, and its metric is given by $g_{i j}=u(x) \delta_{i j}$, then the conformal factor satisfies the partial differential

*Received February 26, 2008; accepted for publication June 13, 2008. The authors are pleased to acknowledge funding provided in part by Natural Sciences and Engineering Research Council of Canada Grants 311685-05 (AB) and 217006-03 (RJM), United States National Science Foundation Grant DMS-0354729 (RJM), and an NSERC Undergraduate Student Research Award held at the University of Toronto (AS).

${ }^{\dagger}$ Department of Mathematics, University of Toronto, Toronto, Ontario M5S 2E4, Canada (almut @math.toronto.ca).

${ }^{\ddagger}$ Department of Mathematics, University of Toronto, Toronto, Ontario M5S 2E4, Canada (mccann @math.toronto.edu).

$\S$ Department of Mathematics, Stanford University, Stanford, CA 94305, USA (asmith3@math. stanford.edu). 
equation

$$
\frac{\partial u}{\partial t}=\Delta \log u+\frac{n-2}{4}|\nabla \log u|^{2} .
$$

Like the Ricci flow, which also describes the evolution of the metric by a curvaturedriven nonlinear heat equation, the Yamabe flow expands regions of negative curvature while contracting regions of positive curvature. In the special case of a two-manifold, the curvature term $R g_{i j}$ on the right hand side of equation (1) is just twice the Ricci curvature, and the Yamabe flow agrees with the Ricci flow. An important difference in dimensions $n>2$ is that the Yamabe flow preserves the conformal class of the metric, while the Ricci flow generally does not.

On a compact manifold, the average value $\bar{R}$ of the scalar curvature determines whether the total volume increases $(\bar{R}<0)$ or decreases $(\bar{R}>0)$ under the Yamabe flow. In particular, a sphere, where $\bar{R}$ is positive, collapses after a finite time. One usually normalizes the flow by setting

$$
\frac{\partial g_{i j}}{d s}=-\frac{1}{n-1}(R-\bar{R}(s)) g_{i j}
$$

which amounts to rescaling the solutions of (1) so that the volume of the manifold is held constant, and reparametrizing time. The normalized Yamabe flow starting from an arbitrary smooth initial metric on a compact connected manifold is immortal, in the sense that it exists for all positive times and never develops singularities. For large classes of initial metrics, in particular for every conformally flat metric, it converges to a solution of the Yamabe problem, i.e., a metric of constant curvature in the conformal class of the initial metric $[12,4,33,24,3]$.

Less is known about the Yamabe flow on non-compact manifolds. For smooth complete conformally flat metrics on $\mathbf{R}^{2}$ that have uniformly bounded curvature and finite width (as defined in [6]), the Ricci flow has a unique solution. As $t \rightarrow \infty$ it converges to the so-called cigar soliton $[31,32,15,16]$, which is the only eternal solution in that class [6]. The cigar soliton is a fixed point of the flow that has everywhere positive, radially decreasing curvature. For the Yamabe flow on noncompact manifolds in dimension $n>2$, solutions are guaranteed to exist at least for a short time, if the Ricci curvature is initially bounded below [20].

Our starting point is a family of closed-form solutions to the porous medium equation

$$
\frac{\partial v}{\partial t}=\frac{1}{m} \Delta\left(v^{m}\right)
$$

that was first discovered some twenty years ago by Titov and Ustinov [26] and Tar$\operatorname{tar}[25]$ (see [30]), and later rediscovered independently several times [19, 22, 9]. They are relevant for the Yamabe flow, because solutions of equation (2) in dimension $n>2$ are transformed into positive solutions of equation (4) with $m=\frac{n-2}{n+2}$ by setting $u=v^{\frac{4}{n+2}}$. For equation (2), the solutions look like

$$
u(x, t)=\frac{1}{a(t)+\langle x, P(t) x\rangle},
$$

where $a(t)$ is a positive real-valued function and $P(t)$ is a diagonal matrix with positive entries. We will use the detailed analysis of these solutions by Denzler and McCann [9]. 
In two dimensions, equation (2) reduces to the log-diffusion equation

$$
\frac{\partial u}{\partial t}=\Delta \log u
$$

which can also be formally derived from the porous medium equation (4) by differentiating at $m=0$. The analysis of Denzler and McCann [9] does not directly apply to the log-diffusion equation in two dimensions, but the quadratic ansatz in equation (5) remains valid, and again leads to a system of ordinary differential equations that can be solved by quadratures.

We are interested in the geometric evolution of the cigar-shaped manifolds that correspond to these closed-form solutions of the porous medium equation. The rotationally symmetric solutions in the family are the Barenblatt profiles, also known as self-similar solutions since they evolve by time-dependent homothety. In the case of the log-diffusion equation in two dimensions, the Barenblatt profile gives rise to the cigar soliton mentioned above. In each dimension $n>2$, it produces an analogue of the cigar soliton that collapses in finite time. However, most of the solutions in the family are not rotationally symmetric and evolve not by self-similar scaling and dilation but rather by a more complicated affine self-similarity. Below we describe how asymmetric cigar-shaped manifolds converge to the cigar solitons.

An interesting question that we do not address is to what extent the special solutions considered here model more general solutions of the porous medium equation. The exponent $m=\frac{n-2}{n+2}$ that is relevant for the Yamabe flow in $n>2$ dimensions lies in the fast-diffusion regime $0<m<1$, where compactly supported initial data immediately develop everywhere positive tails. The Cauchy problem for the fast-diffusion equation is well-posed in $L_{l o c}^{1}$ if the initial data are given by a measure that is not too concentrated on small sets [21]. The behavior of finitemass solutions is well understood in the range $1-\frac{2}{n}<m<1$, where mass is conserved (see $[28,18,2]$ and the references there). For $m=\frac{n-2}{n+2}$, which lies below this range, it is known that solutions of finite mass vanish identically after a finite time, and that their asymptotic behavior is governed by a rotationally symmetric solution with profile $v \sim\left(1+|x|^{2}\right)^{-\frac{n+2}{2}}[10,8,29]$. In contrast, the closed-form solutions of $[26,25,19,22,9]$, including the Barenblatt profile $v \sim\left(1+|x|^{2}\right)^{-\frac{n+2}{4}}$, have infinite mass.

The Cauchy problem for the log-diffusion equation in dimension $n=2$ is not wellposed even for radially symmetric smooth positive data. Solutions with initial values dominated by $(|x| \log |x|)^{-2}$ and solutions in $L^{1} \cap L^{p}$ are non-unique and become identically zero in finite time [17]. Finite-mass solutions can be determined uniquely by imposing boundary conditions at infinity that fix the flux [27], which is a geometric quantity related to the total curvature of the corresponding manifold. On the other hand, if the initial condition is sufficiently positive to be non-integrable then the solution is unique [17]. In particular, a solution constructed under the quadratic ansatz in equation (5) uniquely solves the corresponding initial-value problem. The Barenblatt profile attracts large classes of non-negative solutions with infinite mass [15, 16].

2. Statement of the results. By a cigar manifold we denote a conformally flat manifold that can be parametrized over $\mathbf{R}^{n}(n \geq 2)$ with a conformal factor of the form

$$
u(x)=\frac{1}{a+\langle v, x\rangle+\langle x, P x\rangle},
$$


where $a$ is a positive constant, $v$ is a vector in $\mathbf{R}^{n}$, and $P$ is a positive definite symmetric matrix. The cigar manifold is symmetric or round if $P$ is a multiple of the identity matrix, and asymmetric or fluted otherwise. The standard cigar is the conformally flat manifold that can be parametrized over $\mathbf{R}^{n}$ with

$$
u(x)=\frac{1}{1+|x|^{2}} .
$$

One can replace the constant $a$ in equation (7) with 1 by scaling the coordinates, remove the linear part by a translation, and diagonalize the quadratic form by a rotation in such a way that the eigenvalues of $P$ appear in increasing order. These coordinate changes represent isometries of the manifold. Dilating the manifold by a factor $\lambda$ amounts to replacing $P$ with $\lambda^{-2} P$. Two cigar manifolds are isometric, if and only if the corresponding quadratic forms have the same eigenvalues with the same multiplicities.

In two dimensions, every cigar manifold is homothetic to $M_{p}=\left(\mathbf{R}^{2}, g_{i j}=u_{p} \delta_{i j}\right)$ for some $p \in(0,1]$. The parameter $p$ determines the shape of the manifold through the conformal factor

$$
u_{p}(x, y)=\frac{1}{1+p x^{2}+p^{-1} y^{2}} .
$$

Specifically, equation (7) describes $\lambda M_{p}$, where $\lambda=(\operatorname{det} P)^{-1 / 4}$, and $p$ is the smaller eigenvalue of the matrix $(\operatorname{det} P)^{-1 / 2} P$.

For each value of $p \in(0,1], M_{p}$ is complete and has infinite area. In polar coordinates $(x, y)=e^{\rho}(\cos \theta, \sin \theta)$, the end of $M_{p}$ is asymptotic to the cylinder $C_{p}=$ $\left(\mathbf{R} \times \mathbf{S}^{1}, g_{i j}=w_{p} \delta_{i j}\right)$, with the conformal factor given by

$$
w_{p}(\theta)=\frac{1}{p \cos ^{2} \theta+p^{-1} \sin ^{2} \theta} .
$$

If $p<1$, then $C_{p}$ is symmetric under translations along its axis but not under rotations, and contains two infinite strips of negative curvature. In $M_{p}$, the region of negative curvature consists of two lobes that stretch from the cylindrical end towards the center of the cap, where the curvature assumes its maximum. As $p$ approaches 1, the variation of the curvature decreases and the lobes recede. The limiting case $M_{1}$ is the standard cigar. It is symmetric under a one-parameter group of rotations, has everywhere positive, radially decreasing curvature, and its end is asymptotic to the flat cylinder $C_{1}=\left(\mathbf{R} \times \mathbf{S}^{1}, \delta_{i j}\right)$.

The cigar soliton and its dilates, given by $\lambda M_{1}$ for any $\lambda>0$, are steady-state solutions of the two-dimensional Ricci flow. Our first result provides a closed-form expression for non-constant solutions $M_{p(t)}$, which shows that the family of twodimensional cigar manifolds is preserved by the Ricci flow.

THEOREM 1 (Ricci flow of asymmetric cigars). Consider the Ricci flow starting at time $t=0$ from the two-dimensional fluted cigar manifold $\lambda M_{p_{0}}$ whose size and shape are determined by $\lambda>0$ and $p_{0} \in(0,1)$. Set $t_{0}=-\frac{1}{2} \tanh ^{-1}\left(p_{0}\right)<0$ and define

$$
p(t)=\tanh \left(2\left(t-t_{0}\right)\right) .
$$

Then $\lambda M_{p\left(\lambda^{-2} t\right)}$ solves the Ricci flow for all $t>\lambda^{2} t_{0}$. 
This solution is immortal but not ancient, in the sense that it exists for all positive times but cannot be followed beyond time $t_{\alpha}=\lambda^{2} t_{0}$ into the past. As $t \rightarrow \infty$, we see that $p(t) \rightarrow 1$, and hence the solution approaches a multiple of the cigar soliton.

Different topologies have been used in the literature to define the convergence of manifolds. In the spirit of [7], we consider two manifolds to be close if there exists a biLipschitz map between them whose Lipschitz constant is close to one. The Lipschitz distance between two metric spaces $M$ and $N$ is defined by

$$
d_{\text {Lip }}(M, N)=\inf _{f: M \rightarrow N} \log \max \left\{\operatorname{Lip}(f), \operatorname{Lip}\left(f^{-1}\right)\right\},
$$

where the infimum extends over all bi-Lipschitz maps $f: M \rightarrow N$, and $\operatorname{Lip}(f)$ denotes the Lipschitz constant of $f$.

Similar to the Gromov-Hausdorff distance, this defines a metric on isometry classes of abstract metric spaces (see $[13,23]$ ). If $M$ and $N$ are dilated simultaneously by the same factor $\lambda$, the Lipschitz distance remains unchanged, while the Gromov-Hausdorff distance is multiplied by $\lambda$. By definition, $d_{L i p}(M, N)<\infty$ if $M$ and $N$ are bi-Lipschitz equivalent, and $d_{L i p}(M, N)=0$ if and only if they are isometric. The triangle inequality is satisfied, because the composition of Lipschitz maps is again Lipschitz, with a constant no larger than the product of the individual Lipschitz constants.

Our second result describes the rate at which $M_{p(t)}$ converges to $M_{1}$.

Theorem 2 (Rate of convergence of a fluted cigar to the standard cigar). Let $p_{0} \in(0,1)$ be given, and consider the Ricci flow $M_{p(t)}$ that starts at time $t=0$ from the two-dimensional asymmetric cigar manifold $M_{p_{0}}$, as described in Theorem 1. Then $M_{p(t)}$ converges exponentially to the standard cigar $M_{1}$ as $t \rightarrow \infty$, and

$$
\frac{b}{4} e^{-4 t} \leq d_{L i p}\left(M_{p(t)}, M_{1}\right)+O\left(e^{-8 t}\right) \leq b e^{-4 t}
$$

holds with $b=\frac{1-p_{0}}{1+p_{0}}$.

Since the Lipschitz distance between bounded metric spaces dominates the Gromov-Hausdorff distance (see, for example, $[13,23]$ ), it follows that $M_{p(t)}$ converges exponentially to $M_{1}$ with respect to the Gromov-Hausdorff metric on compact subsets.

We have formulated Theorem 2 for the Lipschitz distance because the standard parametrizations of the cigar manifolds provide convenient bi-Lipschitz maps between them. This allows us to bound the Lipschitz distance from above by the relative error between the conformal factors, which was introduced by Vázquez in [28]. The difference is that the Lipschitz distance does not distinguish between isometric spaces. For the Gromov-Hausdorff metric, the lack of compactness causes technical issues. Although the Gromov-Hausdorff distance from any cigar manifold $\lambda M_{p}$ to $M_{1}$ is finite, it is not clear to us whether it converges to zero as $p \rightarrow 1$. It is also not easy to bound the Gromov-Hausdorff distance from below, because it is defined as an infimum over the large set of isometric embeddings into abstract metric spaces. Similar considerations apply to the notion of convergence defined in Chapter 8 of [7].

The theorem suggests, but does not imply, that there should be a constant $b^{*} \in$ $\left[\frac{b}{4}, b\right]$ such that $d_{L i p}\left(M_{p(t)}, M_{I}\right)=b^{*} e^{-4 t}+O\left(e^{-8 t}\right)$. It is interesting to speculate on whether the symmetrizing flow of the asymmetric cigar models the convergence of a more general class of solutions to the standard cigar. For perturbations of the 
conformal factor which depend on angle only, linearization of the Yamabe flow around the standard cylinder suggests that the fluted cylinder converges with the second slowest mode. The slowest mode involves only one oscillation in the amplitude of the curvature around the circumference of the cylinder, instead of two. This leads us to conjecture that any conformally Euclidean manifold whose single end is isometric or asymptotic to a translation-invariant cylinder, will converge to the cigar soliton at least as quickly as our asymmetric cigars do, provided only that the cross-section of the asymptotic cylinder possesses an isometric involution to exclude the slowest mode.

In higher dimensions $n \geq 2$, every cigar manifold can be parametrized over $\mathbf{R}^{n}$ with a conformal factor

$$
u_{P}(x)=\frac{1}{1+\sum_{i=1}^{n} p_{i} x_{i}^{2}},
$$

where $P$ is a diagonal matrix whose entries $0<p_{1} \leq \ldots \leq p_{n}$ determine the size and the shape of the manifold $M_{P}=\left(\mathbf{R}^{n}, u_{P} \delta_{i j}\right)$. The end of $M_{P}$ is asymptotic to a cylinder $C_{P}=\left(\mathbf{R} \times \mathbf{S}^{n-1}, g_{i j}=w_{P} \delta_{i j}\right)$, with conformal factor

$$
w_{P}(\sigma)=\frac{1}{\sum_{i=1}^{n} p_{i} \phi_{i}(\sigma)^{2}},
$$

where $\phi$ is the standard isometric embedding of $\mathbf{S}^{n-1}$ into $\mathbf{R}^{n}$. The standard cylinder $C_{I}$ has constant scalar curvature given by $4(n-1)(n-2)$. Two cigar manifolds are isometric, if and only if their matrices agree; they are homothetic with $M_{P}=\lambda M_{Q}$, if and only if $P=\lambda^{-2} Q$.

As in two dimensions, the family of cigar manifolds is preserved by the Yamabe flow. Closed-form solutions $M_{P(t)}$ in $n>2$ dimensions are provided by positive solutions of the porous medium equation (4) within the family of $[26,25,19,22,9]$. These solutions exist on a maximal time interval that depends on the initial value of $P$. They can be ancient, but collapse at some finite time $t_{\omega}<\infty$. The diameter of the sphere at infinity shrinks asymptotically with $\left(t_{\omega}-t\right)^{1 / 2}$, while the shape of the manifold approaches a thin round cigar.

To emphasize the analogy with two dimensions, we avoid the collapse by rescaling the flow so that it conserves the Riemannian $(n-1)$-surface area of the sphere at infinity, as defined by the conformal factor in equation (11). The normalized flow satisfies equation (3), where $\bar{R}(s)$ is the average of the scalar curvature of $M_{Q(s)}$ over the sphere at infinity with respect to this Riemannian measure. The rescaling slows down the evolution, so that $s \rightarrow \infty$ logarithmically as $t \rightarrow t_{\omega}$. The standard cigar $M_{I}$ is a time-invariant solution of the normalized flow, and asymmetric cigar manifolds converge exponentially to $M_{I}$.

Theorem 3 (Rate at which normalized Yamabe flow symmetrizes a fluted cigar). Fix $n \geq 2$, and let $Q_{0}$ be $a n \times n$ diagonal matrix with positive entries that satisfies

$$
\int_{\mathbf{S}^{n-1}} w_{Q_{0}}(\sigma)^{\frac{n-1}{2}} d \sigma=\left|\mathbf{S}^{n-1}\right| .
$$

Let $M_{Q(s)}$ be a solution of the normalized Yamabe flow in equation (3), where $\bar{R}(s)$ is the average of the scalar curvature of $M_{Q(s)}$ over the sphere at infinity with respect to the Riemannian measure defined by the conformal factor $w_{Q(s)}$. If $M_{Q(s)}$ starts from 
$M_{Q_{0}}$ at time $s=0$, then

$$
\frac{b}{2 n(n-1)} e^{-(n+2) s} \leq d_{L i p}\left(M_{Q(s)}, M_{I}\right)+O\left(e^{-2(n+2) s}\right) \leq b e^{-(n+2) s}
$$

as $s \rightarrow \infty$ with some constant $b>0$ that depends only on $Q_{0}$.

We note that the surface area of the sphere at infinity in a cigar manifold can be characterized intrinsically as the value of the isoperimetric profile of the manifold, i.e., the minimal surface area that is needed to enclose a subset of given Riemannian $n$ volume, in the limit where the volume goes to infinity. In two dimensions, this agrees with the aperture defined in [31,32] and is closely related with the width defined in [6].

In dimension $n=2$, the cigar soliton is a steady-state solution of both equation (1) and the normalized equation (3). Moreover, the rate of convergence given by Theorem 2 agrees to leading order with the rate of convergence for the normalized flow in Theorem 3. The reason is that under the two-dimensional Ricci flow, the length of the loop at infinity in $M_{p(t)}$ converges exponentially to $2 \pi$, and hence as $t \rightarrow \infty$ the solution of equation (1) is asymptotic, up to a constant time-shift, to the solution of equation (3) where the length of this loop has been normalized to $2 \pi$.

3. Ricci flow on two-dimensional asymmetric cigars. We use the method of [9] to construct the Ricci flow on two-dimensional cigar manifolds. If $u$ solves equation (6), then $\pi=\frac{1}{u}$ satisfies

$$
\frac{\partial \pi}{\partial t}=\pi \Delta \pi-|\nabla \pi|^{2} .
$$

In the context of the porous medium equation, $-\pi$ plays the role of the pressure. We look for positive solutions in two dimensions that are quadratic in $x$,

$$
\pi(x, y, t)=a(t)+(x, y) P(t)(x, y)^{t} .
$$

Here, $a(t)$ is a positive function, and $P(t)$ is a $2 \times 2$ diagonal matrix with positive entries. This ansatz results in the matrix equation

$$
\frac{d P}{d t}=2(\operatorname{tr} P) P-4 P^{2},
$$

coupled with a differential equation for $a(t)$ that is geometrically irrelevant for the Ricci flow. Thus the Ricci flow preserves the set of two-dimensional cigar manifolds.

Proof of Theorem 1. Let $P(t)$ be a diagonal $2 \times 2$ matrix with positive entries $p(t) \leq q(t)$ that solves equation (12). The first line of Eq. (12) reads

$$
\frac{d p}{d t}=2\left(\operatorname{det} P-p^{2}\right) .
$$

The key observation is that $\operatorname{det} P$ is constant,

$$
\frac{d}{d t} \log \operatorname{det} P=\operatorname{tr}\left\{P^{-1} \frac{d P}{d t}\right\}=0 .
$$

The parameter $\lambda=\operatorname{det} P^{-1 / 4}$ determines the size of the corresponding asymmetric cigar manifold. Scaling the solution by $\tilde{P}(t)=\lambda^{2} P\left(\lambda^{2} t\right)$, we consider only $\operatorname{det} P=1$ and $p \in(0,1]$. The constant solution $p(t) \equiv 1$ of equation (13) corresponds to the 
cigar soliton. For $0<p<1$, the solution is given by $p(t)=\tanh \left(2\left(t-t_{0}\right)\right)$, where $t_{0}$ is determined by the initial value of $p$. By construction, $\lambda M_{p\left(\lambda^{-2} t\right)}$ solves the Ricci flow with initial value $\lambda M_{p_{0}}$.

We turn to the bi-Lipschitz estimates claimed in Theorem 2. It is easy to bound the Lipschitz distance between two cigar manifolds from above:

LEMma 1 (Maximum bi-Lipschitz distance of a fluted cigar to the standard cigar). For any value of $p \in(0,1]$,

$$
d_{\text {Lip }}\left(M_{p}, M_{1}\right) \leq \frac{1}{2}|\log p| .
$$

Proof. The identity map on $\mathbf{R}^{2}$ defines a map from $M_{p}$ to $M_{1}$ via the standard coordinate charts. To estimate its Lipschitz constant $L$, consider the ratio

$$
\frac{u_{1}(x, y)}{u_{p}(x, y)}=\frac{1+p x^{2}+p^{-1} y^{2}}{1+x^{2}+y^{2}} \in\left[p, p^{-1}\right] .
$$

This means that tangent vectors at any point $(x, y) \in \mathbf{R}^{2}$ are dilated by factors that lie between $p^{1 / 2}$ and $p^{-1 / 2}$, and thus

$$
d_{\text {Lip }}\left(M_{p}, M_{1}\right) \leq \log L \leq \frac{1}{2}|\log p| .
$$

The complementary inequality requires a lower bound on the Lipschitz constants of all bi-Lipschitz maps from $M_{p}$ to $M_{1}$ as $p \rightarrow 1$. We simplify this problem by restricting the maps to the cylindrical ends $C_{p}$ and $C_{1}$. The next lemma shows that the distance between any two cigar manifolds is at least as large as the distance between their cylindrical ends.

Lemma 2 (Cigars are at least as dissimilar as their ends). For any choice of $0<p, q \leq 1$ and $\lambda, \mu>0$,

$$
d_{\text {Lip }}\left(\lambda M_{p}, \mu M_{q}\right) \geq d_{\text {Lip }}\left(\lambda C_{p}, \mu C_{q}\right) .
$$

Proof. Let $f: \lambda M_{p} \rightarrow \mu M_{q}$ be a bi-Lipschitz map with Lipschitz constant $L$. We will construct a bi-Lipschitz map $g: \lambda C_{p} \rightarrow \mu C_{q}$ by conjugating $f$ near the cylindrical end with auxiliary functions $\psi_{k}$, adding a suitable translation, and passing to a limit.

In polar coordinates on $M_{p}$, the conformal factor from equation (8) becomes

$$
\tilde{u}_{p}(\rho, \theta)=\frac{1}{e^{-2 \rho}+p \cos ^{2} \theta+p^{-1} \sin ^{2} \theta} .
$$

On the annulus in $C_{p}$ that corresponds to parameter values $(\rho, \theta) \in[-k, k] \times \mathbf{S}^{1}$, define a map into $M_{p}$ by $\psi_{k}(\rho, \theta)=e^{2 k+\rho}(\cos \theta, \sin \theta)$. The same formula also defines a bijection $\tilde{\psi}_{k}: \mu C_{q} \longrightarrow \mu M_{q} \backslash\left\{0_{q}\right\}$, where $0_{q}$ denotes the point corresponding to the origin $(x, y)=(0,0)$ in $\mu M_{q}$. If $k$ is large, then the image of $\psi_{k}$ is an annulus far up in the cylindrical end of $M_{p}$. Comparing equation (14) with equation (9), we see that $\psi_{k}$ is bi-Lipschitz with constant bounded by $1+O\left(e^{-2 k}\right)$ as $k \rightarrow \infty$. This remains true if we dilate both $M_{p}$ and $C_{p}$ by $\lambda$. 
Let $O_{p} \in \lambda M_{p}$ be the point corresponding to $(x, y)=(0,0)$ and denote the distance from $f^{-1}\left(O_{q}\right)$ to $0_{p}$ in $\lambda M_{p}$ by $\delta$. Since the distance $d_{k}=\lambda p^{1 / 2} \sinh ^{-1}\left(p^{1 / 2} e^{k}\right)$ of the image of $\psi_{k}$ from $O_{p}$ grows without bound as $k \rightarrow \infty$, taking $k$ large enough ensures $d_{k}>\delta$. It then follows from the Lipschitz continuity of $f^{-1}$ that the image of $f \circ \psi_{k}$ does not intersect a ball of radius $\left(d_{k}-\delta\right) / L$ about $O_{q}$. On the complement of this ball, the bi-Lipschitz constant of $\tilde{\psi}_{k}^{-1}$ is bounded by $1+O\left(e^{-2\left(d_{k}-\delta\right) / L}\right)$.

To complete the construction, we choose a translation $\tau_{k}$ along the $\rho$-direction in $\mu C_{q}$ such that $g_{k}=\tau_{k} \circ \tilde{\psi}_{k}^{-1} \circ f \circ \psi_{k}$ maps the point corresponding to $(\rho, \theta)=(0,0)$ in $\lambda C_{p}$ to a point on the loop parametrized by $\rho=0$ in $\mu C_{q}$. This ensures that the maps $g_{k}$ are pointwise bounded. Then $g_{k}$ is bi-Lipschitz, with constant bounded by $L+O\left(e^{-2 k}+e^{-2\left(d_{k}-\delta\right) / L}\right)$ as $k \rightarrow \infty$. On any compact subset of $\lambda C_{p}$, the coordinate functions of $g_{k}$ are defined for $k$ sufficiently large, and the Arzelà-Ascoli theorem allows us to choose a subsequence $g_{k_{j}}$ that converges locally uniformly to a limiting function $g: \lambda C_{p} \rightarrow \mu C_{q}$. Clearly, $g$ is bi-Lipschitz with constant at most $L$.

Finally, we estimate from below the bi-Lipschitz distance of a slightly fluted cylinder to a round one.

Lemma 3 (Bi-Lipschitz distance of a slightly fluted cylinder to a round one). As $p \rightarrow 1$,

$$
\inf _{\lambda>0} d_{L i p}\left(C_{p}, \lambda C_{1}\right) \geq \frac{1}{8}|\log p|+O(p-1)^{2} .
$$

Proof. Consider an annulus $A \subset C_{p}$ that corresponds under polar coordinates to $(\rho, \theta) \in[0, r] \times \mathbf{S}^{1}$. The area of the annulus is given by

$$
v(r)=\int_{0}^{r} \int_{0}^{2 \pi} \frac{1}{p \cos ^{2} \theta+p^{-1} \sin ^{2} \theta} d \theta d \rho=r \cdot\left(2 \pi+O(p-1)^{2}\right) .
$$

Since the conformal factor $w_{p}$ in equation (9) does not depend on $\rho$, the loops parametrized by $\rho=$ const. are geodesics, and have length

$$
c=\int_{0}^{2 \pi}\left(\frac{1}{p \cos ^{2} \theta+p^{-1} \sin ^{2} \theta}\right)^{1 / 2} d \theta=2 \pi+O(p-1)^{2}
$$

as $p \rightarrow 1$. We have used that the first order term in the Taylor expansion of $c$ about $p=1$ vanishes because the value of the integral does not change if $p$ is switched with $p^{-1}$. The shortest geodesics that join the upper with the lower boundary of $A$ occur at $\theta=\pi / 2$ and $\theta=3 \pi / 2$ and have length

$$
h(r)=r \cdot p^{1 / 2} .
$$

It follows that the distance between any pair of points on the upper and lower boundary of the annulus cannot exceed $h(r)+c$.

Let now $g$ be a bi-Lipschitz map from $C_{p}$ to $\lambda C_{1}$, and let $L$ be its Lipschitz constant. Then $g(A)$ is topologically an annulus, which is bounded by non-contractible loops in $\lambda C_{1}$. Since $g$ is Lipschitz continuous, these loops have length at most $L \cdot c$, and the distance between any pair of points on upper and lower loop is bounded by $L \cdot(h(r)+c)$. It follows that the area of $g(A)$ in the flat cylinder $\lambda C_{1}$ is bounded by $L^{2} \cdot c \cdot(h(r)+c)$. Since $g^{-1}$ is also Lipschitz continuous, the area of $A$ satisfies

$$
v(r) \leq L^{4} \cdot c \cdot(h(r)+c) .
$$


By taking $r \rightarrow \infty$, this forces

$$
L^{4} \geq \frac{v(r)}{c \cdot h(r)}=p^{-1 / 2}\left(1+O(p-1)^{2}\right),
$$

and we conclude that

$$
\inf _{\lambda>0} d_{L i p}\left(C_{p}, \lambda C_{1}\right) \geq \log L \geq \frac{1}{8}|\log p|+O(p-1)^{2} .
$$

Proof of Theorem 2. We insert the explicit solution from Theorem 1 into Lemma 1 and into Lemmas 2 and 3 with $\lambda=\mu=1$. Since $\tanh \left(2\left(t-t_{0}\right)\right)=1-2 e^{-4\left(t-t_{0}\right)}+$ $O\left(e^{-8 t}\right)$ as $t \rightarrow \infty$, and $e^{4 t_{0}}=\frac{1-p_{0}}{1+p_{0}}$, the theorem is proved.

We remark that the proof of Theorem 2 did not take full advantage of Lemma 3, since we fixed the dilation factor to be $\lambda=1$. Together with Theorem 2, Lemma 3 shows that

$$
\left.\inf _{\lambda>0} d_{L i p}\left(M_{p(t)}, \lambda M_{1}\right) \geq \frac{1}{4} d_{L i p}\left(M_{p(t)}, M_{1}\right)\right)\left(1+O\left(e^{-4 t}\right)\right)
$$

as $t \rightarrow \infty$. This means that the solution $\left\{M_{p(t)}\right\}_{t>0}$ meets the curve of round cigars $\left\{\lambda M_{1}\right\}_{\lambda>0}$ at the point $\lambda=1$ transversally, avoiding a cone of opening angle $\sin ^{-1}\left(\frac{1}{4}\right)$ whose axis is tangent to the curve.

4. Yamabe flow on cigar manifolds in dimension $n>2$. We next describe the closed-form solutions of the porous medium equation (4) that were constructed in $[26,25,9]$. We specialize the derivation of Denzler-McCann to the case $m=\frac{n-2}{n+2}$, $\gamma=-\frac{n+2}{4}$, and choose different normalizations constants and sign conventions.

We write the partial differential equation (2) in terms of the function $\pi=\frac{1}{u}$ as

$$
\frac{\partial \pi}{\partial t}=\pi \Delta \pi-\frac{n+2}{4}|\nabla \pi|^{2},
$$

see the pressure formulation of the porous medium equation in equation (3) of [9]. The quadratic ansatz $\pi(x)=a(t)+\langle x, P(t) x\rangle$ yields

$$
\frac{d P}{d t}=2(\operatorname{tr} P) P-(n+2) P^{2}
$$

which agrees with equation (8) of [9] except for a constant factor of $2 \gamma$. Here, $a(t)$ is a positive function that has no relevance for the Yamabe flow, and $P(t)$ is a diagonal matrix with positive entries. According to [9], the general solution of this equation is given by

$$
P(t)=\operatorname{det}(B+\tau I)^{\frac{2}{n+2}}(B+\tau I)^{-1}, \quad \frac{d t}{d \tau}=\frac{1}{n+2} \operatorname{det}(B+\tau I)^{\frac{-2}{n+2}},
$$

where $B$ is a diagonal matrix with $\operatorname{tr} B=0$, and $\tau=\tau(t)$ is an increasing real-valued function defined on a maximal interval $\left(t_{\alpha}, t_{\omega}\right)$. The entries of $B$ and the function $\tau$ are determined by the initial conditions. The value of $t_{\alpha}$ may be finite or infinite, but $t_{\omega}$ is always finite, and

$$
\tau(t)=\left((n-2)\left(t_{\omega}-t\right)\right)^{-\frac{n+2}{n-2}}+O\left(t_{\omega}-t\right)^{\frac{n+2}{n-2}},
$$


see equation (16) in [9]. Setting

$$
\lambda(t)=\left((n-2)\left(t_{\omega}-t\right)\right)^{1 / 2},
$$

shows that

$$
P(t)=\lambda(t)^{-2}\left\{I-\left((n-2)\left(t_{\omega}-t\right)\right)^{\frac{n+2}{n-2}} B+O\left(t_{\omega}-t\right)^{2 \frac{n+2}{n-2}}\right\}
$$

as $t \rightarrow t_{\omega}$. The case $B=0$ gives the symmetric solutions $P(t)=\lambda(t)^{-2} I$.

The proof of Theorem 3 also requires bounds on the distance from an asymmetric cigar manifold to the standard cigar. The upper bound from Lemma 1 extends immediately to higher dimensions:

Lemma 1'. (Maximum bi-Lipschitz distance of a fluted cigar to the standard cigar). Let $n \geq 2$. For any diagonal matrix $P$ with entries $0<p_{1} \leq \cdots \leq p_{n}$,

$$
d_{\text {Lip }}\left(M_{P}, M_{I}\right) \leq \frac{1}{2} \max \left\{\left|\log p_{1}\right|,\left|\log p_{n}\right|\right\} .
$$

For the complementary lower bound, we consider the cylindrical end of $M_{P}$. Let $\phi$ be the standard isometric embedding of $\mathbf{S}^{n-1}$ into $\mathbf{R}^{n}$. In polar coordinates $x=e^{\rho} \phi(\sigma)$ on $M_{P}$, the conformal factor $u_{P}$ from equation (10) becomes

$$
\tilde{u}_{P}(\rho, \sigma)=\frac{1}{e^{-2 \rho}+\sum_{i=1}^{n} p_{i} \phi_{i}(\sigma)^{2}} .
$$

The distance between cigar manifolds is bounded by the distance between their cylindrical ends also in higher dimensions:

Lemma 2'. (Cigars are at least as dissimilar as their ends). For $n \geq 2$,

$$
d_{L i p}\left(M_{P}, M_{Q}\right) \geq d_{L i p}\left(C_{P}, C_{Q}\right) .
$$

The lower bound on the distance between cylinder manifolds is a bit more involved than for $n=2$ :

Lemma 3'. (Bi-Lipschitz distance of a slightly fluted cylinder to a round one). Let $n \geq 2$. For any diagonal matrix $P$ with entries $0<p_{1} \leq \cdots \leq p_{n}$,

$$
\inf _{\lambda>0} d_{L i p}\left(C_{P}, \lambda C_{I}\right) \geq \frac{1}{4 n} \log \frac{p_{n}}{\frac{1}{n} \sum_{i=1}^{n} p_{i}} .
$$

Proof. Let $g: C_{P} \rightarrow \lambda C_{I}$ be a map with bi-Lipschitz constant $L$. As in the proof of Lemma 3, we apply $g$ to an annulus $A \subset C_{P}$ that is parametrized by coordinates $(\rho, \sigma) \in[0, r] \times \mathbf{S}^{n-1}$. The shortest geodesics joining the two boundary spheres have length

$$
h(r)=r \cdot p_{n}^{-1 / 2} .
$$


The Riemannian $(n-1)$-surface area of the cross section of $A$ is given by

$$
c=\int_{\mathbf{S}^{n-1}} w_{P}(\sigma)^{\frac{n-1}{2}} d \sigma
$$

where the integration is with respect to the standard measure on $\mathbf{S}^{n-1}$. For the $n$-volume of $A$ we compute

$$
v(r)=r \int_{\mathbf{S}^{n-1}} w_{P}(\sigma)^{\frac{n}{2}} d \sigma
$$

The last two quantities are related by Jensen's inequality $\frac{v(r)}{r \cdot\left|\mathbf{S}^{n-1}\right|} \geq\left(\frac{c}{\left|\mathbf{S}^{n-1}\right|}\right)^{\frac{n}{n-1}}$.

We argue as in the proof of Lemma 3 that for large $r$

$$
L^{2 n} \geq \frac{v(r)}{c \cdot h(r)} \geq \frac{r}{h(r)}\left(\frac{c}{\left|\mathbf{S}^{n-1}\right|}\right)^{\frac{1}{n-1}} .
$$

Another application of Jensen's inequality brings us to

$$
L^{2 n} \geq p_{n}^{1 / 2}\left(\frac{1}{\left|\mathbf{S}^{n-1}\right|} \int_{\mathbf{S}^{n-1}} \sum_{i=1}^{n} p_{i} \phi_{i}(\sigma)^{2} d \sigma\right)^{-\frac{1}{2}}=\left(\frac{p_{n}}{\frac{1}{n} \sum_{i=1}^{n} p_{i}}\right)^{\frac{1}{2}} .
$$

The claim follows upon taking logarithms.

The next lemma shows that under the Yamabe flow in $n>2$ dimensions, the shape of a cigar manifold approaches the shape of a round cigar at least twice as fast as the width of the cigar is collapsing, and much faster than that in low dimensions.

LEMma 4 (Rate of symmetrization of a fluted cigar collapsing under Yamabe flow). Consider a solution $M_{P(t)}$ of the Yamabe flow in dimension $n>2$, where $P(t)$ is a diagonal matrix with entries $0<p_{1}(t) \leq \cdots \leq p_{n}(t)$. Let $t_{\omega}$ be the time of collapse of $M_{P(t)}$, and define $\lambda(t)$ as in equation (16). There exists a constant $b_{0}>0$ such that

$$
\frac{b_{0}}{2 n(n-1)}\left(t_{\omega}-t\right)^{\frac{n+2}{n-2}} \leq d_{L i p}\left(M_{P(t)}, \lambda(t) M_{I}\right)+O\left(t_{\omega}-t\right)^{2 \frac{n+2}{n-2}} \leq b_{0}\left(t_{\omega}-t\right)^{\frac{n+2}{n-2}}
$$

as $t \rightarrow t_{\omega}$.

Proof. The function $P(t)$ is given by equation (17), where $B$ is traceless and diagonal with entries $b_{1} \geq \cdots \geq b_{n}$. It follows from Lemma 1 ' that

$$
\begin{aligned}
d_{\text {Lip }}\left(M_{P(t)}, \lambda(t) M_{I}\right) & =d_{\text {Lip }}\left(\lambda(t)^{-1} M_{P(t)}, M_{I}\right) \\
& \leq \frac{1}{2} \max \left\{\left|\log \left(\lambda(t)^{2} p_{1}(t)\right)\right|,\left|\log \left(\lambda^{2}(t) p_{n}(t)\right)\right|\right\} \\
& =\frac{1}{2} \max \left\{\left|b_{1}\right|,\left|b_{n}\right|\right\}\left((n-2)\left(t_{\omega}-t\right)\right)^{\frac{n+2}{n-2}}+O\left(t_{\omega}-t\right)^{2 \frac{n+2}{n-2}} .
\end{aligned}
$$

Similarly, Lemmas 2' and 3' imply

$$
\begin{aligned}
d_{\text {Lip }}\left(M_{P(t)}, \lambda(t) M_{I}\right) & \geq \inf _{\lambda} d_{L i p}\left(C_{P(t)}, \lambda C_{I}\right) \\
& \geq \frac{1}{4 n} \log \frac{p_{n}(t)}{\frac{1}{n} \sum_{i=1}^{n} p_{i}(t)} \\
& =\frac{1}{4 n}\left|b_{n}\right|\left((n-2)\left(t_{\omega}-t\right)\right)^{\frac{n+2}{n-2}}+O\left(t_{\omega}-t\right)^{2 \frac{n+2}{n-2}} .
\end{aligned}
$$


Since $\sum b_{i}=0$, we must have $\left|b_{1}\right| \leq(n-1)\left|b_{n}\right|$, and the claim follows by setting $b_{0}=\frac{(n-1)}{2}(n-2)^{\frac{n+2}{n-2}}\left|b_{n}\right|$.

5. Normalized Yamabe flow on cigar manifolds. Let $P$ be a diagonal matrix with entries $0<p_{1} \leq \cdots \leq p_{n}$, and denote the Riemannian $(n-1)$-surface area of the cross section of $M_{P}$ at infinity by

$$
c_{P}=\int_{\mathbf{S}^{n-1}} w_{P}(\sigma)^{\frac{n-1}{2}} d \sigma .
$$

Note that $c_{P}$ is positive homogeneous of degree $(1-n) / 2$, and its gradient with respect to $\left(p_{1}, \ldots, p_{n}\right)$ is non-vanishing.

Assume that $M_{P(t)}$ solves the Yamabe flow in equation (1), i.e., $P(t)$ solves equation (15). Let $P_{0}=P(0)$ be the initial value of $P$. Using equation (1) in the form $\frac{\partial w}{\partial t}=-\frac{1}{n-1} R w$, the chain rule yields

$$
\frac{d}{d t} c_{P(t)}=-\frac{1}{2} \int_{\mathbf{S}^{n-1}} R_{P(t)}(\sigma) w_{P(t)}^{\frac{n-1}{2}}(\sigma) d \sigma
$$

Normalizing the cross section to its initial value and adjusting the speed accordingly,

$$
Q(s)=\left(\frac{c_{P(t)}}{c_{P_{0}}}\right)^{\frac{2}{n-1}} P(t), \quad \frac{d s}{d t}=\left(\frac{c_{P(t)}}{c_{P_{0}}}\right)^{\frac{-2}{n-1}},
$$

we see that $c_{Q(s)} \equiv c_{P_{0}}$, and $M_{Q(s)}$ solves the normalized Yamabe flow in equation (3) with

$$
\bar{R}(s)=\frac{1}{c_{P_{0}}} \int_{\mathbf{S}^{n-1}} R_{Q(s)}(\sigma) w_{Q(s)}^{\frac{n-1}{2}} d \sigma
$$

by the chain rule. Equation (15) transforms into

$$
\frac{d Q}{d s}=f(Q) Q-(n+2) Q^{2}
$$

where $f$ is a real-valued function given by $f(Q)=2 \operatorname{tr} Q-\frac{\bar{R}(s)}{n-1}$.

Heuristically, we would like to explain the precise exponential rate of convergence of $M_{Q(s)}$ to the cigar soliton that is claimed in Theorem 3 by linearizing equation (19) about $Q=I$. Since $f$ is smooth away from $Q=0$, positive homogeneous of degree one, and symmetric in the eigenvalues $q_{1}, \ldots q_{n}$ of $Q$, its Taylor expansion takes the form

$$
f(Q)=\frac{(n+2)}{n} \operatorname{tr} Q+O(Q-I)^{2}
$$

as $Q \rightarrow I$. Inserting this back into equation (19) gives

$$
\frac{d Q}{d s}=(n+2)\left\{\frac{1}{n}(\operatorname{tr} Q) I-Q\right\}+O(Q-I)^{2} .
$$

It follows that the linearization of equation (19) about $Q=I$ has a simple eigenvalue 0 (associated with the dilation invariance) and an eigenvalue $-(n+2)$ of multiplicity $(n-1)$ (governing the dynamics on the hypersurface $\left.c_{Q}=\left|\mathbf{S}^{n-1}\right|\right)$. By Lemmas 1', 2', and 3', this yields the exponential rate of convergence claimed in Theorem 3, but 
without the error estimate. We will obtain the error estimate by rescaling the closedform solutions of Denzler-McCann [9].

Proof of Theorem 3. Consider first the case $n>2$. Assume that $Q_{0}$ is a diagonal matrix with positive entries such that $c_{Q_{0}}=\left|\mathbf{S}^{n-1}\right|$. Let $Q(s)$ be a solution of equation (19) with $Q(0)=Q_{0}$. If $P(t)$ is the corresponding solution of equation (15) with $P(0)=Q_{0}$, then

$$
d_{L i p}\left(M_{Q(s)}, M_{I}\right)=d_{L i p}\left(M_{P(t)},\left(\frac{c_{P(t)}}{\left|\mathbf{S}^{n-1}\right|}\right)^{\frac{1}{n-1}} M_{I}\right) .
$$

We will replace the dilation factor on the right hand side by $\lambda(t)$ and control the error with the triangle inequality. Using the scaling properties of $c_{P}$ and the fact that the linear term in its Taylor expansion about $P=I$ is proportional to $\operatorname{tr}(P-I)$, we obtain from the expression for $P(t)$ in equation (17)

$$
c_{P(t)}=\lambda(t)^{(n-1)}\left\{\left|\mathbf{S}^{n-1}\right|+O\left(t_{\omega}-t\right)^{2 \frac{n+2}{n-2}}\right\} .
$$

It follows that

$$
d_{\text {Lip }}\left(M_{Q(s)}, M_{I}\right)=d_{\text {Lip }}\left(M_{P(t)}, \lambda(t) M_{I}\right)+O\left(\left(t_{\omega}-t\right)^{2 \frac{n+2}{n-2}}\right)
$$

as $t \rightarrow t_{\omega}$. Lemma 4 bounds the right hand side from above and below by constant multiples of $\left(t_{\omega}-t\right)^{\frac{n+2}{n-2}}$.

It remains to express the rate of convergence and the errors in terms of the new time variable $s$. We integrate the relationship between the variables in equation (18) to obtain for $t<t_{\omega}$

$$
\begin{aligned}
s & =\int_{0}^{t} \lambda(t)^{-2}\left\{1+O\left(t_{\omega}-t\right)^{2 \frac{n+2}{n-2}}\right\} d x \\
& =s_{0}-\frac{1}{(n-2)} \log \left(t_{\omega}-t\right)+O\left(\left(t_{\omega}-t\right)^{2 \frac{n+2}{n-2}}\right) .
\end{aligned}
$$

The claim follows by solving

$$
t_{\omega}-t=e^{-(n-2)\left(s-s_{0}\right)}\left\{1+O\left(e^{-2(n+2) s}\right)\right\},
$$

and applying Lemma 4.

If $n=2$, let $p_{0}$ be the smaller eigenvalue of $\left(\operatorname{det} Q_{0}\right)^{-1 / 2} Q_{0}$, and compare $M_{Q(s)}$ with the solution $M_{p(t)}$ of equation (1) given by Theorem 1 . The loop at infinity in $M_{p(t)}$ has length

$$
2 \pi\left\{1+O(1-p(t))^{2}\right\}=2 \pi+O\left(e^{-8 t}\right) .
$$

In this case, the relationship in equation (18) implies

$$
Q(s)=P(t)\left\{1+O\left(e^{-8 t}\right)\right\}, \quad s=s_{0}+t+O\left(e^{-8 t}\right),
$$

and the claim follows from Theorem 1. 
[1] P. Bénilan And M. G. Crandall, The continuous dependence on $\phi$ of solutions of $u_{t}=\Delta \phi(u)$, Indiana U. Math. J., 30 (1981), pp. 161-177.

[2] M. Bonforte And J. L. VÁzQUez, Global positivity estimates and Harnack inequalities for the fast diffusion equation, J. Funct. Anal., 240 (2006), pp. 399-428.

[3] S. Brendue, Convergence of the Yamabe flow for arbitrary initial energy, J. Diff. Geom., 69 (2005), pp. 217-278.

[4] B. CHOw, The Yamabe flow on conformally flat manifolds with positive Ricci curvature, Comm. Pure Appl. Math., 45 (1992), pp. 1003-1014.

[5] B. Chow And D. Knopf, The Ricci flow: an introduction. Mathematical Surveys and Monographs, 110. American Mathematical Society, Providence, RI, 2004.

[6] P. Daskalopoulos and N. Sesum, Eternal solutions to the Ricci flow on $\mathbf{R}^{2}$, Intern. Mat. Res. Notices, 2006 (2006), \# 83610.

[7] G. David and S. Semmes, Fractured fractals and broken dreams, Chapter 8, (Oxford University Press, 1997.)

[8] M. Del Pino AND M. SÁEz, On the extinction profile for solutions of $u_{t}=\Delta u^{(N-2) /(N+2)}$, Indiana Univ. Math. J., 50 (2001), pp. 611-628.

[9] J. Denzler and R. J. MCCann, Nonlinear diffusion from a delocalized source: affine selfsimilarity, time reversal $\&$ nonradial focusing geometries, Ann. Inst. H. Poincaré Anal. Non Linéaire, 25 (2008), pp. 865-888.

[10] V. A. Galaktionov and L. A. Peletier, Asymptotic behaviour near finite-time extinction for the fast diffusion equation, Arch. Rational Mech. Anal., 139 (1997), pp. 83-98.

[11] R. S. Hamilton, The Ricci flow on surfaces, Contemp. Math., 71 (1988), pp. 237-262.

[12] R. S. Hamilton, (unpublished note on Yamabe flow, credited in [4]).

[13] J. Heinonen, Lectures on Analysis on Metric Spaces, (Springer Verlag, 2000)

[14] M. A. Herrero And M. Pierre, The Cauchy problem for $u_{t}=\Delta u^{m}$ when $0<m<1$, Trans. Amer. Math. Soc., 291 (1985), pp. 145-158.

[15] S.-Y. Hsu, Large time behaviour of solutions of the Ricci flow on $\mathbb{R}^{2}$, Pacific Math. J., 197 (2001), pp. 25-41.

[16] S.-Y. Hsu, Asymptotic profile of solutions of a singular diffusion equation as $t \rightarrow \infty$, Nonlinear Anal., 48 (2002), Ser. A: Theory Methods, pp. 781-790.

[17] S.-Y. Hsu, Uniqueness of solutions of a singular diffusion equation, Differential Integral Equations, 16 (2003), pp. 181-200.

[18] Y.-J. Kim And R. J. MCCann, Potential theory and optimal convergence rates in fast nonlinear diffusion, J. Math. Pures Appl., 86 (2006), pp. 42-67.

[19] J. R. KING, Exact multi-dimensional solutions to some nonlinear diffusion equations, Q. J. Mech. Appl. Math., 46 (1993), pp. 419-436.

[20] L. MA AND Y. An, The maximum principle and the Yamabe flow, Partial differential equations and their applications (Wuhan, 1999), pp. 211-224, World Sci. Publ., River Edge, NJ, 1999.

[21] M. Pierre, Nonlinear fast diffusion with measures as data, Nonlinear parabolic equations: qualitative properties of solutions (Rome, 1985), pp. 179-188, Pitman Res. Notes Math. Ser., 149, Longman Sci. Tech., Harlow, 1987.

[22] V. V. Pukhnachev, Exact multi-dimensional solutions of the nonlinear diffusion equation, Journal of Applied Mechanics and Technical Physics, 36 (1995), pp. 169-176.

[23] E. SAUCON, Surface triangulation - the metric approach, arxiv:cs.GR/0401023 (2004 preprint).

[24] H. Schwetlick And M. Struwe, Convergence of the Yamabe flow for "large" energies, J. Reine Angew. Math., 562 (2003), pp. 59-100.

[25] L. TARTAR, Solutions particulières de $u_{t}=\Delta u^{m}$ et comportement asymptotique, (circa 1986, unpublished.)

[26] S. S. Titov And V. A. Ustinov, Investigation of polynomial solutions of the two-dimensional Leibenzon filtration equation with an integer adiabatic exponent, (Russian), in: A.F. Sidorov and S.V. Vershinin (Eds.) Approximate methods for solving boundary value problems of continuum mechanics (Russian), pp. 64-70, 91, Akad. Nauk SSSR, Ural. Nauchn. Tsentr, Sverdlovsk, 1985.

[27] J. L. VÁzquez, J. R. Esteban, and A. Rodriguez, The fast diffusion equation with logarithmic nonlinearity and the evolution of conformal metrics in the plane, Advances in Differential Equations, 1 (1996), pp. 21-51.

[28] J. L. VÁzQUEZ, Asymptotic behaviour for the porous medium equation posed in the whole space. Dedicated to Philippe Bénilan, J. Evol. Eq., 3 (2003), pp. 67-118. Section 21.

[29] J. L. VÁzquez, Smoothing and decay estimates for nonlinear diffusion equations. Equations of porous medium type, Oxford University Press, 2006.

[30] J. L. VÁzQuez, The porous medium equation: mathematical theory, Oxford Mathematical 
Monographs. The Clarendon Press, Oxford 2007.

[31] L.-F. WU, A new result for the porous medium equation derived from the Ricci flow, Bull. Amer. Math. Soc. (N.S.), 28 (1991), pp. 90-94.

[32] L.-F. Wu, The Ricci flow on $\mathbf{R}^{2}$, Communications in Analysis and Geometry, 1 (1993), pp. 439472.

[33] R. YE, Global existence and convergence of the Yamabe flow, J. Diff. Geom., 39 (1994), pp. 3550. 